\title{
Monoamine signaling gene networks unraveled in mouse social stress model
}

\author{
Vladimir Babenko \\ Laboratory of Neuropathology Modeling, Institute of Cytology \\ and Genetics, Siberian Branch of Russian Academy of Sciences, \\ Novosibirsk, Russia \\ bob@bionet.nsc.ru
}

\author{
Natalia Kudryavtseva \\ Laboratory of Neuropathology Mediling Institute of Cytology \\ and Genetics, Siberian Branch of Russian Academy of Sciences, \\ Novosibirsk, Russia \\ natnik@bionet.nsc.ru
}

\section{Research}

Abstract - Synapse signaling manifests the key mechanism of signal transduction across and within brain regions. The advent of RNA-Seq protocol yielded a unique chance to observe stable states of brain region interaction modes based on synapse genes expression profiles. We proposed the subsets of synapse related genes for particular neurotransmitter types, in particular, monoamines (dopamine and serotonin) and glutamate/gaba. These include 3 types: a) vesicular monoamine transporters; b) monoamine transporters; c) synapse receptors.

Upon considering 3 brain regions (dorsal striatum (STR), ventral tegmental area (VTA), and midbrain raphe nuclei (MRN)) we elucidated the specific physiological states of 9 species in the animal stress model published elsewhere (Kudryavtseva et al., 2014) [1].

Keywords - animal stress model, aggression, depression, dorsal striatum, dopamine, serotonin.

\section{Introduction}

The phenomenon of depression and addiction are currently one of the major sources of human disabilities in the world. Their physiology is tightly linked to the limbic system of the striatal system. In particular, addiction was shown to affect the Nucleus accumbens (NAcc) region along with dorsal striatum (STR) in mouse, and caudate/putamen regions in human, also comprising the striatum region. The dorsal striatum maintains supervision of motor activity and stereotypical behaviors. It is involved in cognitive, reward and social hierarchy manifestations. STR exemplifies the initiation and execution of movements via regulation of muscle tone, so it is one of the central brain regions to manifest overall behavior patterns.

The major body of STR cells is Medium Spiny Neurons (MSNs), which account for 95\% of neural cells in STR. Previously, we showed that STR exhibits specific dopamine glutamate alternative signaling [2]. In this work we performed RNA-Seq based analysis in conjunction of other brain regions upon STR, namely, Ventral TGegmental Area (VTA) and Midbrain Raphe Nuclei (MRN), the major sources of dopamine and serotonin, correspondingly.
We compiled the synaptic related genes set to assess the signaling VTA $<->$ STR, MRN $<->$ STR. Next we performed expression profiles in these regions. We elucidated that:

a) Dopamine intake is the highest in aggressive species, and lowest in depressive ones.

b) Dopamine expression in VTA is the highest in certain depressive species, but it's essentially reuptaken back to VTA due to the low D1/D2 receptors density in the depressive STR MSNs.

c) Depressive species uptake serotonin preferably via VTA, while MRN serotonin expression is turned down in their dorsal striatum.

\section{Conclusion}

By means of adapted gene subset we can precisely interpret the signal states in synapses VTA->STR, MRN $>$ STR based on the gene expression profiles. Notably, we identified at least 2 distinct depression - like states in our samples which are featured with distinct gene expression profiles for a range of pathways. It may imply that for certain cases selective serotonin reuptake inhibitor therapy may be non-adequate due to a different etiology of the pathology,

\section{ACKNOWLEDGMENT}

This work is supported by Russian Science Foundation (grant No. 19-15-00026, to NNK)

\section{REFERENCES}

[1] Kudryavtseva N.N., Smagin D.A., Kovalenko I.L., Vishnivetskaya G.B. (2014) Repeated positive fighting experience in male inbred mice. Nature Protocol. 9, 11, 2705 - 2717.

[2] Babenko VN, Galyamina AN, Rogozin IB, Smagin DM' Kudryavtseva NN Dopamine response gene pathways in dorsal striatum MSNs from a gene expression viewpoint: cAMP-mediated gene networks. BMC Neroscience, accepted. Principal Component Analysis on the corresponding 